\title{
Synthesis of a Biological-Based Glycoluril with Phosphorous Acid Tags as a New Nanostructured Catalyst: Application for the Synthesis of Novel Natural Henna-Based Compounds
}

\author{
Saeid Moradi, ${ }^{[a]}$ Mohammad A. Zolfigol, ${ }^{[a]}$ Mahmoud Zarei, ${ }^{*[a]}$ Diego A. Alonso, ${ }^{*[b]}$ Abbas \\ Khoshnood $^{*[b]}$
}

Abstract:

A convenient multicomponent reaction towards the synthesis of natural henna-based 3-methyl-1,4-diphenyl-1,4dihydrobenzo[6,7]chromeno[2,3-c]pyrazole-5,10-dione

derivatives by using a crabby nano glycoluril tetrakis(methylene phosphorous acid) (GTMPA), as an efficient and recyclable catalyst, under neat conditions is described. The major advantages of the synthetic methodology herein described are high yields, short reaction times, and the reusability of the catalyst, which has been fully characterized by Fourier Transform Infrared Spectroscopy (FT-IR), Solid Phosphorus Nuclear Magnetic Resonance ( $\left.{ }^{31} \mathrm{P} N \mathrm{NR}\right)$, Mass spectra (MS), XRay Diffraction (XRD), High Resolution Transmission Electron Microscopy (HRTEM), Scanning Electron Microscope (SEMMapp.), Energy Dispersive X-Ray (EDX), Thermo Gravimetric Analysis (TGA), Differential Thermal Analysis (DTG), Differential Thermal Analysis (DTA) and X-Ray Photoelectron Spectroscopy (XPS). The described biological-based glycoluril with phosphorous acid pending may be applicable for the synthesis of gels, gelators, self-healing and smart hydrogels.

\section{Introduction}

In recent years, there is a great interest in the design and synthesis of easy and safe chemicals and chemical processes. ${ }^{[1-}$ ${ }^{2]}$ Especially the design of new catalysts and catalytic systems from the viewpoint of green chemistry disciplines are more demand. ${ }^{[3-6]}$ Among of catalysts, heterogeneous solid catalysts are promising materials widely used when trying to develop simple catalytic and eco-friendly processes due to their easy recyclability by a simple filtration and/or centrifugation, and the potential for reusing as the most important issues. ${ }^{[7-8]}$ In recent years, researchers are interested in nano-catalysts because of their high specific surface area and hence the high activity and selectivity usually shown by these materials. ${ }^{[9-14]}$ On the other hand, multicomponent reactions (MCRs) have become a very important tool in organic chemistry because three or more starting materials react to form a product, which all or most of the atoms contribute to the final product. ${ }^{[15-17]}$ This type of reactions have been widely used in the synthesis of complex pharmaceutically active heterocyclic compounds, ${ }^{[18-20]}$ especially nitrogen-containing derivatives due to the biological activity usually shown by this type of compounds. ${ }^{[21-23]}$ Functionalized quinone- and naphthoquinone derivatives are molecules often obtained from natural sources (henna's leaves, Streptocapus dunnii,...) which have been widely used as synthetic intermediates in both academia and industry due to their interesting biological and drug-like properties. ${ }^{[24-35]}$ On the other hand, glycoluril as a biological urea based precursor has been used for various purposes. ${ }^{[36]}$

In this paper the synthesis and characterization of a new glycoluril tetrakis(methylene phosphorous acid) (GTMPA)derived solid acid nano-catalyst 2 is reported (Scheme 1). Also, we present an efficient and green 2-catalyzed MCR towards the synthesis of 3-methyl-1,4-diphenyl-1,4dihydrobenzo[6,7]chromeno[2,3-c]pyrazole-5,10-dione derivatives.

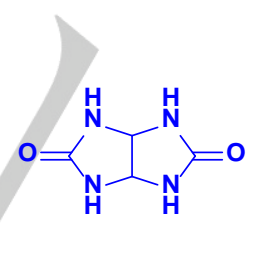

1

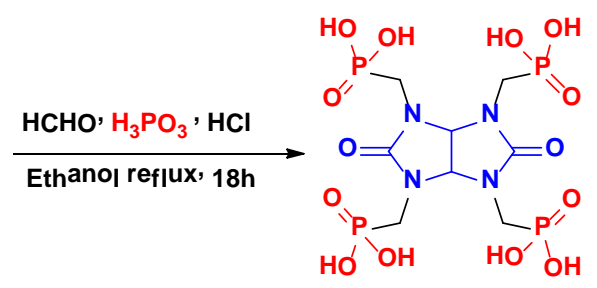

2
Scheme 1. Synthesis of nanostructured catalyst 2. 


\section{Results and Discussion}

Initially, we synthesized the heterogeneous nano-catalyst

2 by a one-step $N$-peralkylation of glycoluril (1) with paraformaldehyde and phosphorous acid in refluxing $\mathrm{EtOH}$ (Scheme 1). This material was characterized using different techniques such as, FT-IR, ${ }^{31} \mathrm{P}$ NMR, Mass spectra, X-ray diffraction patterns (XRD), scanning electron microscopy (SEM) with elemental mapping and EDX, high resolution transmission electron microscopy (HRTEM), thermogravimetry (TG), derivative thermogravimetry (DTG) analysis, differential thermal analysis (DTA), and X-ray photoelectronic spectroscopy (XPS) (Figure 1-8)

As shown in Fig. 1 the FT-IR spectrum of 2 showed an absorption band at $3200 \mathrm{~cm}^{-1}$ corresponding to the $\mathrm{OH}$ stretching frequency of the phosphorous acid. Furthermore, the absorption band at $1678 \mathrm{~cm}^{-1}$ certified the presence of the amide carbonyl moiety and peaks related to $\mathrm{P}=\mathrm{O}, \mathrm{C}-\mathrm{P}$ and $\mathrm{C}-\mathrm{N}$ bond were observed at 1219, 1110 and $991 \mathrm{~cm}^{-1}$ respectively (Figure $1)$.

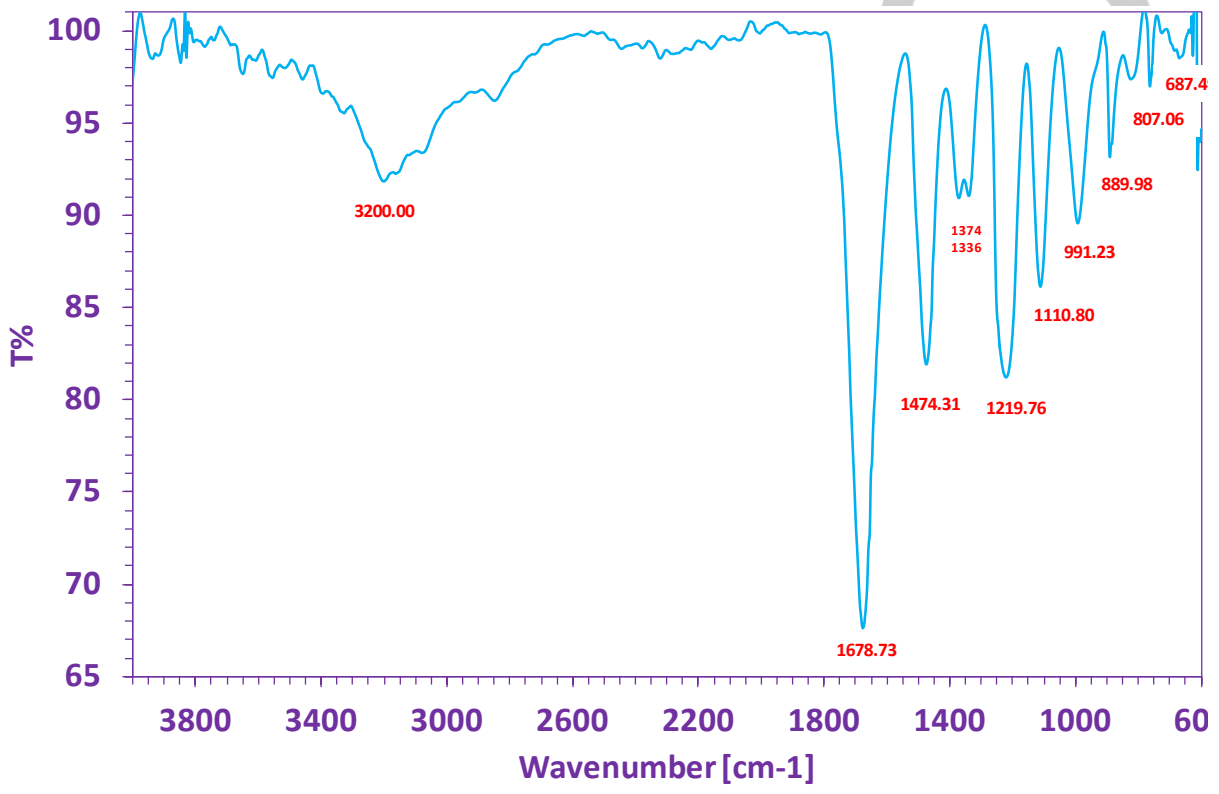

Fig. 1. FT-IR spectrum of GTMPA

On the other hand, the solid ${ }^{31} \mathrm{P}$ NMR spectrum of 2 showed the signal of the hydrogen-bonded $\mathrm{P}-\mathrm{OH}$ protons as a single resonance at $10.74 \mathrm{ppm}$, confirming the presence of the phosphorous acid moiety in the catalyst (Figure 2), white solid, M. P: $>300{ }^{\circ} \mathrm{C}$ and Mass spectra of 2 showed MS $\mathrm{m} / \mathrm{z}(\%): 519$ $\left(\mathrm{M}^{+}, 1\right)$ (Figure 3).

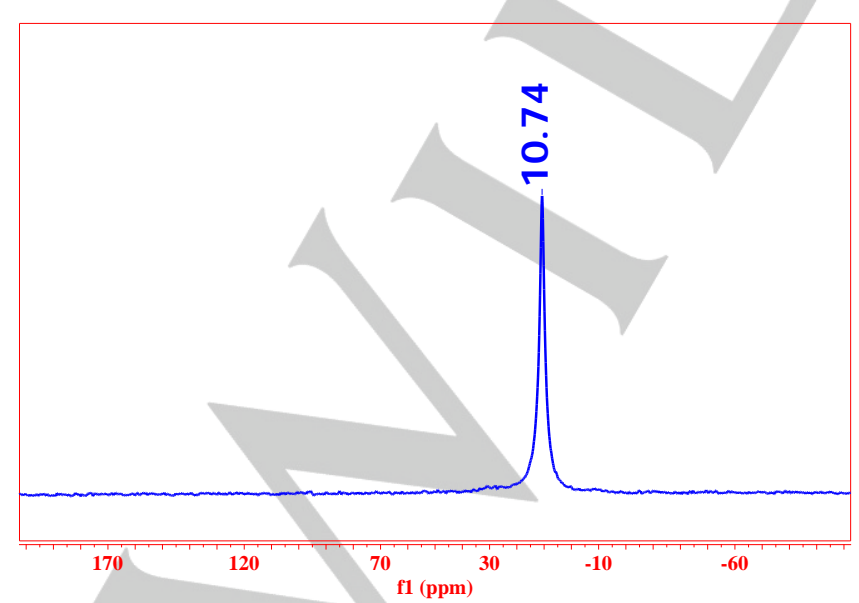

Fig. 2. ${ }^{31} \mathrm{P}$ NMR spectrum (solid state, $202 \mathrm{MHz}$ ) of catalyst 2.
The X-ray diffraction (XRD) pattern of the catalyst was considered in a domain of 5 to 70 degree. Peak width (FWHM), size and inter planer distance related to XRD pattern of $\mathbf{2}$ were

investigated in the 17.1 to 38.3 degree (Fig. 4 ). The obtained data is collected in Table 1 . The crystallite size $D$ of the solid catalyst was calculated using the Debye-Sherrer formula $D$ $=\mathrm{K} \lambda /(\beta \operatorname{Cos} \theta)$, with $\lambda$ being the $X$-ray wavelength $(\lambda=0.154 \mathrm{~nm})$, $K$ is the Scherrer constant (0.9), $B$ the peak width of halfmaximum (FWHM), and $\theta$ is the Bragg diffraction angle. The interplanar distance was calculated via the Bragg equation: dhkl $=\lambda /(2 \sin \theta),(\lambda:$ Cu radiation $(0.154178 \mathrm{~nm})$. Finally, the crystal dimensions from various diffraction lines using the Scherrer equation were found to be in the nanometer range (29.06 $47.31 \mathrm{~nm})$. 


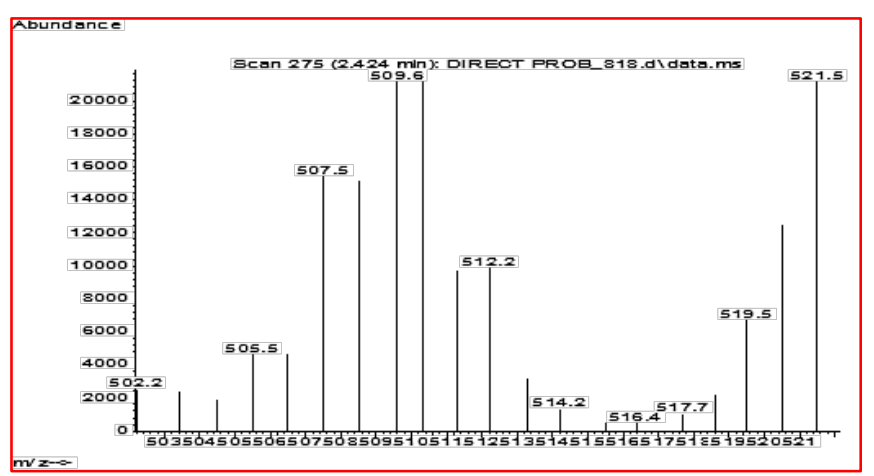

Fig. 3. Mass spectra of GTMTPA

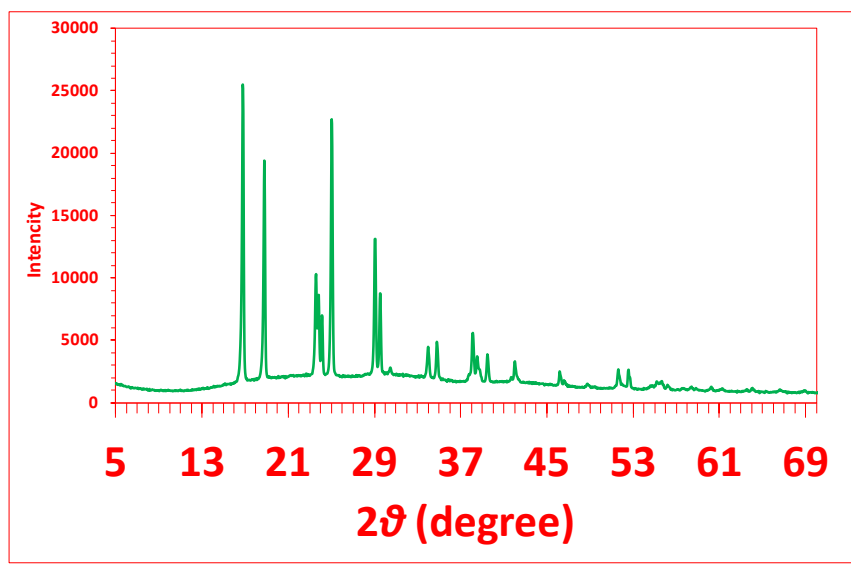

Fig. 4. XRD pattern of 2 .

Table 1. X-ray diffraction (XRD) data of GTMPA.

\begin{tabular}{lcccc}
\hline Entry & $2 \theta$ & $\begin{array}{c}\text { Peak width } \\
\text { (degree) } \\
\end{array}$ & Size $[\mathrm{nm}]$ & $\begin{array}{c}\text { Inter planar } \\
\text { distance } \\
\text { (FWH) }\end{array}$ \\
\hline
\end{tabular}

\begin{tabular}{ccccc}
\hline 1 & 17.1 & 0.2 & 40.16 & 0.51851 \\
2 & 19.1 & 0.3 & 26.87 & 0.46465 \\
3 & 23.9 & 0.3 & 27.09 & 0.37231 \\
4 & 25.3 & 0.3 & 27.16 & 0.35201 \\
5 & 29.3 & 0.3 & 27.39 & 0.30480 \\
6 & 35 & 0.3 & 27.79 & 0.25636 \\
7 & 38.3 & 0.5 & 16.82 & 0.23410 \\
\hline \multicolumn{5}{r}{ The surface typical textural and morphological properties }
\end{tabular}
of the catalyst and the difference in adhesion and dispersion can be clearly studied from the HRTEM, SAED, SEM and elemental SEM-mapping images as exposed in Fig. 5a-h. Initially, we used high-resolution transmission electron microscopy (HRTEM) to study the detailed structure of the solid catalyst 2 . Figures $5 \mathrm{a}$ and $b$ show the HRTEM image of $\mathbf{2}$ supported on a TEM copper grid. Also, the combined selected area electron diffraction (SAED) patterns of the catalyst are shown in Figure $5 \mathrm{c}$. The HRTEM images of the catalyst showed near spherical nanoparticles morphology with an appropriate mono disparity. Moreover, we also analyzed the morphology of catalyst $\mathbf{2}$ using scanning electron microscopy (SEM). As depicted in Figure $5 d$ for the SEM micrograph, and Figures $5 \mathrm{e}-\mathrm{h}$ for the SEMelemental mapping $(\mathrm{C}, \mathrm{N}, \mathrm{O}$ and $\mathrm{P})$, the presence of $\mathrm{C}, \mathrm{N}, \mathrm{O}$ and $P$ elements in the catalyst with a good distribution over the catalyst surface was verified. 


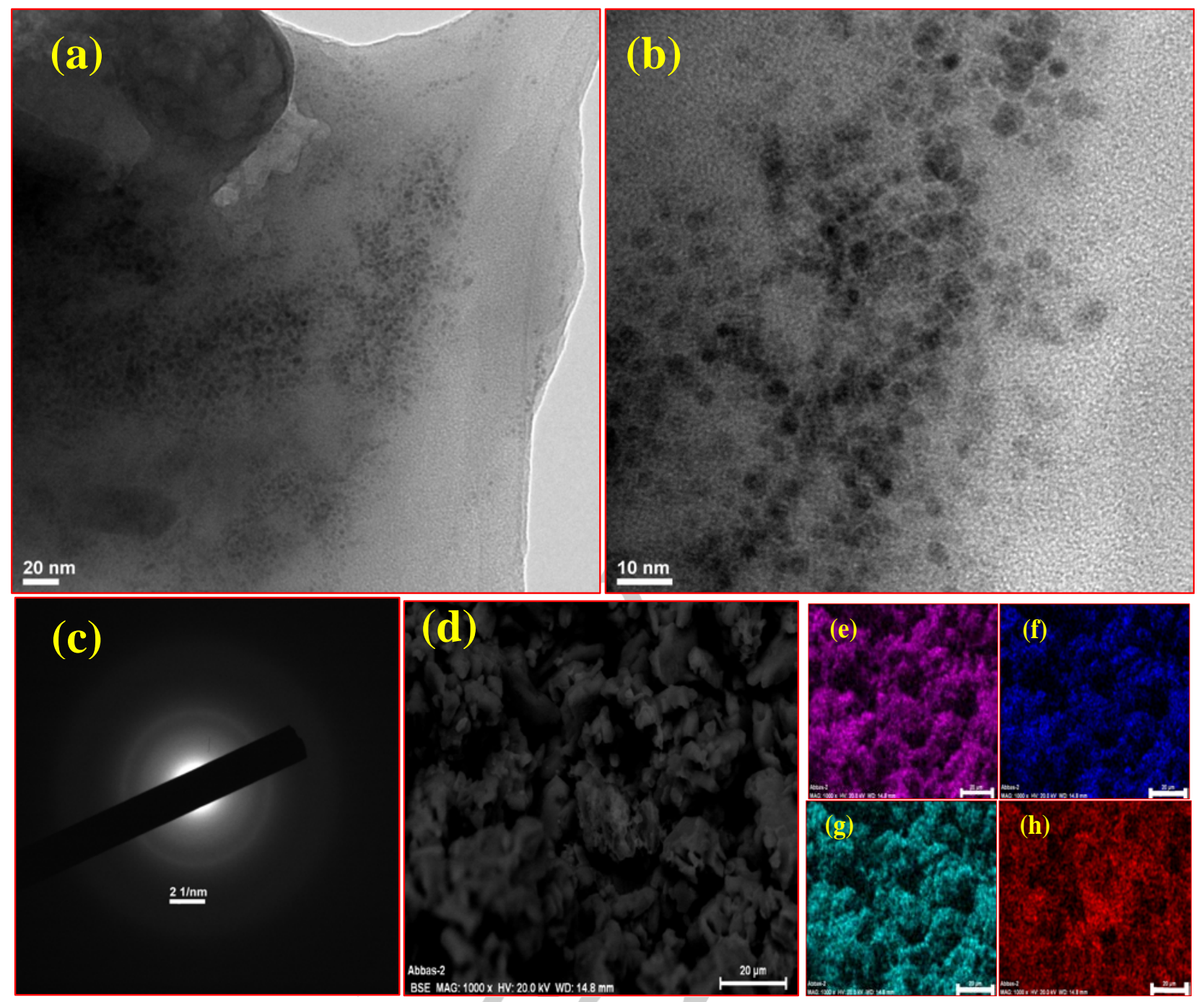

Fig. 5. High resolution transmission electron microscopy (HRTEM) (a-b), selected area electron diffraction (SAED) (c), scanning electron microscopy (SEM) of glycoluril (d); elemental maps (EDX) of $\mathrm{N}(\mathrm{e}) ; \mathrm{C}(\mathrm{f}) ; \mathrm{P}(\mathrm{g})$ and $\mathrm{O}(\mathrm{h})$ atoms for catalyst 2.

Fig. 6 collects representative energy-dispersive X-ray spectroscopy (EDS) elemental distribution images of catalyst $\mathbf{2}$, which corroborated the existence of phosphorus on the catalyst structure in a $\sim 2 \mathrm{wt} \%$. The elemental analysis also showed a 43 $w t \%$ of carbon, a 29 wt $\%$ of nitrogen and a 26 wt $\%$ of oxygen.

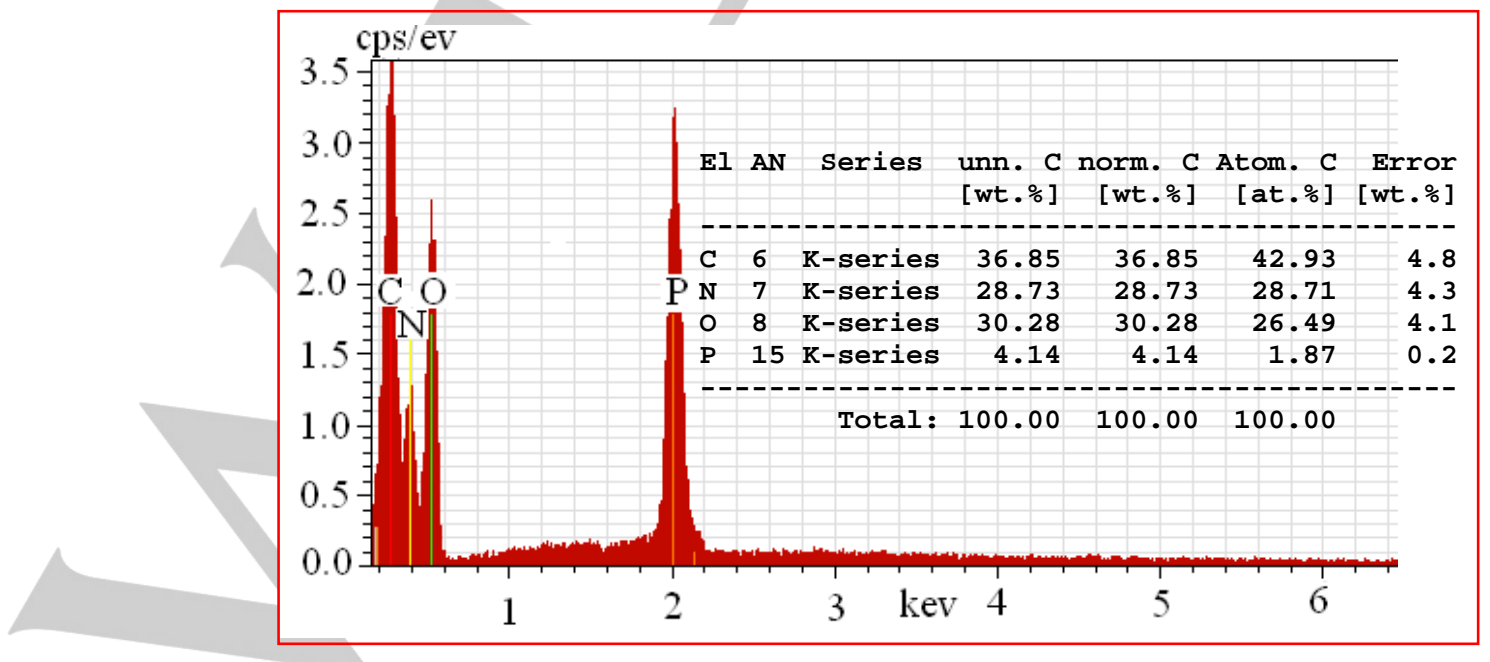

For internal use, please do not delete. Submitted_Manuscript 
Fig. 6. Energy-Dispersive X-ray spectroscopy (EDS) analysis of 2.

In order to monitor the thermal stability and behavior of the GTMPA-derived catalyst, the thermogravimetric analysis (TGA), derivative thermogravimetric analysis (DTG), as well as the differential thermal analysis (DTA) were conducted (Fig. 7 a and b). Regarding TGA, a first weight loss stage, related to the removal of surface-adsorbed water and organic solvents, took place between 25 and $100{ }^{\circ} \mathrm{C}$, involving a weight loss of $2.2 \%$.
The main weight loss, associated with the decomposition of the catalyst, took place around $200-400{ }^{\circ} \mathrm{C}$ in a single exothermic step manner and involved a weight loss of 39.5\%. Moreover, the DTA analysis diagram showed a general negative downward slope between $25{ }^{\circ} \mathrm{C}$ to $700{ }^{\circ} \mathrm{C}$ in which, decomposition of catalyst and its precursor in nitrogen atmosphere were exothermic (Fig. 7b).

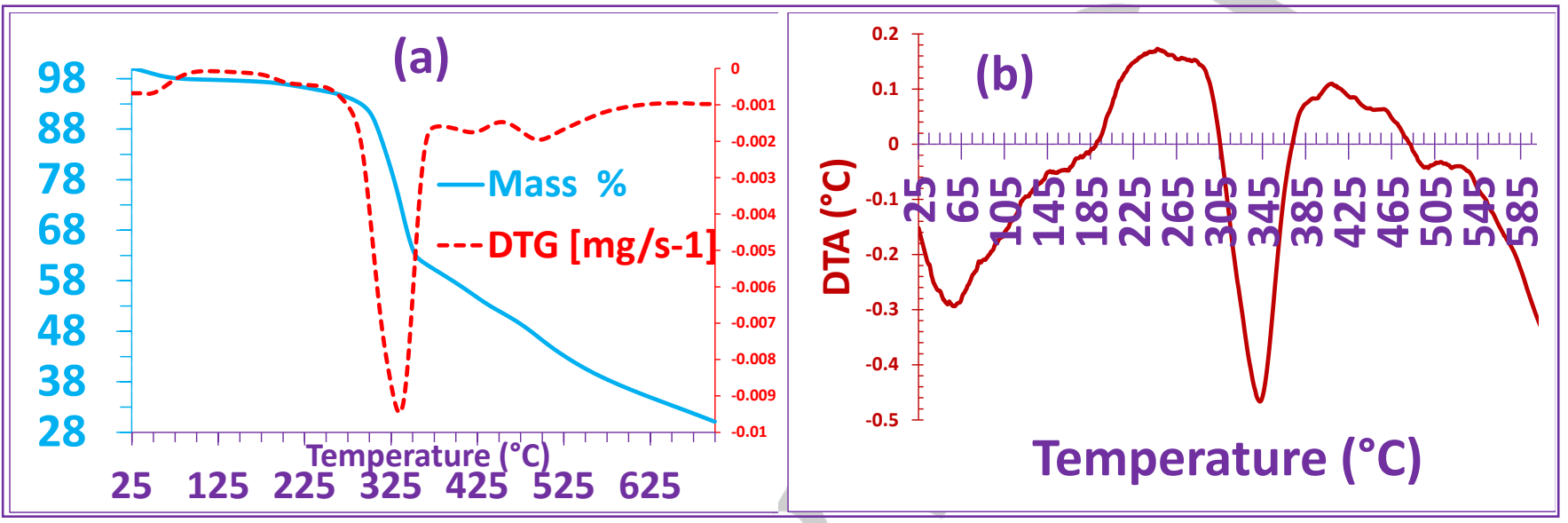

Fig. 7. Thermal stability and behavior of 2: (a) thermogravimetric analysis (TGA) and derivative thermogravimetric analysis (DTG); (b) differential thermal analysis (DTA).

Additional studies with X-ray photoelectron spectra (XPS) were carried out to analyze the surface character and chemical composition of the prepared catalyst 2. As expected, the peaks corresponding to N1s, O1s, C1s, and P2p were clearly seen in the XPS survey spectrum of catalyst 2 , thus confirming the presence of the phosphonomethyl $\left(-\mathrm{CH}_{2} \mathrm{PO}_{3} \mathrm{H}_{2}\right)$ moiety covalently bonded to 1 (Figure 8a). As shown in Figure 8b, the phosphorus peaks located around $133.68 \mathrm{eV}$ could be fitted into two $2 \mathrm{p}$ peaks located at 133.45 (C-P-O) and $134.35 \quad(\mathrm{C}-\mathrm{P}=\mathrm{O})$ eV corresponding to 2 p3/2 and 2 p1/2 level, respectively in an area ratio $1 / 0.5$. Moreover, the C1s XPS spectrum of glycoluril could be deconvoluted into three peaks at 284.62, 287.08, 288.73 eV fitted with a broad peak at $285.70 \mathrm{eV}$. These energies confirmed the existence of $\mathrm{CH}-\mathrm{CH}, \mathrm{N}-\mathrm{CH}_{2}-\mathrm{P}, \mathrm{N}-\mathrm{C}=\mathrm{O}$ and $\mathrm{N}-\mathrm{CH}-\mathrm{N}$ moieties, respectively in and area ratio of $0.7 / 1 / 1 / 0.2$ and an atomic percentage of $11.7 / 15.9 / 15.8 / 3.2$. From the same atomic percentage and area ratio showed by the groups $\mathrm{C}=\mathrm{O}$ and $\mathrm{C}-\mathrm{P}$, we could reasoned that the surface of catalysts was principally covered by these two carbon moieties. On the other hand, the symmetrical N1s XPS spectrum of 2, located around $399.68 \mathrm{eV}$, could be deconvoluted into one peak at $399.66 \mathrm{eV}$ which revealed the existence of $\mathrm{N}-\mathrm{C}=\mathrm{O}$. This peak should be fitted with a broader peak (higher FWHM) with a $0.01 \mathrm{eV}$ binging energy difference at $400.66 \mathrm{eV}$ in an area ratio of $1 / 0.1$. $^{\text {[3] }}$ This second broad peak suggests the presence of secondary amides in the catalyst. Finally, the unsymmetrical oxygen peaks located around 531.48 eV could be fitted into two 1s peaks located at $531.37(\mathrm{C}-\mathrm{P}=\mathrm{O})$ and $532.71(\mathrm{~N}-\mathrm{C}=\mathrm{O}) \mathrm{eV}$ in an area ratio of $1 / 0.8$. ${ }^{[38]}$ 

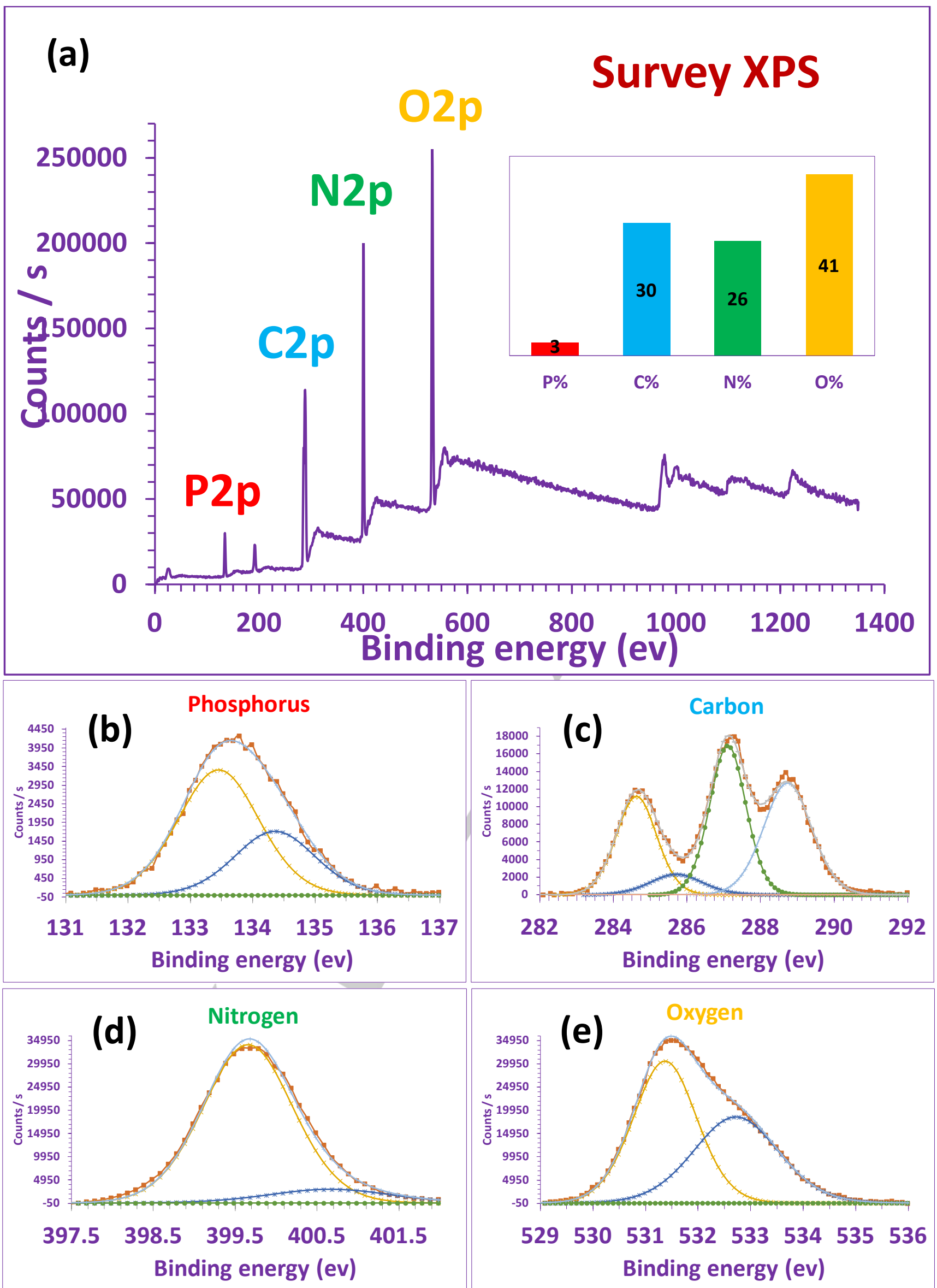

For internal use, please do not delete. Submitted_Manuscript 
Fig. 8. X-ray photoelectron spectra (XPS) survey spectrum (a); and high resolution XPS spectra of P2p (b); C1s (c); N1s (d); and O1s (e) of GTMPA

After characterization of catalyst $\mathbf{2}$, we studied its catalytic activity as solid acid in the multicomponent synthesis of 3-methyl1,4-diphenyl-1,4-dihydrobenzo[6,7]chromeno[2,3-c]pyrazole-5,10dione derivatives. In order to optimize the reaction conditions, the condensation reaction of benzaldehyde, 2-hydroxynaphthalene1,4-dione and 3-methyl-1-phenyl-1H-pyrazol-5(4H)-one to obtain compound $\mathbf{3 a}$ was chosen as a model reaction. Initially, optimization of the catalyst loading and reaction temperature was carried out under neat conditions (Table 2). As shown, low yields were obtained in the absence of catalyst both at 25 and $100{ }^{\circ} \mathrm{C}$
(Table 2, entries 1 and 2). At $100^{\circ} \mathrm{C}$, the optimal catalyst loading was $5 \mathrm{~mol} \%$ (Table 2, entries 3-6). Regarding temperature, the process was still highly efficient even working at $50{ }^{\circ} \mathrm{C}$ using 5 mol\% of 2 (Table 2, entry 8 ). Regarding solvent optimization, the model reaction (Scheme 1 ) was carried out using various solvents (EtOAc, $\mathrm{CH}_{3} \mathrm{CN}$, EtOH and $\mathrm{H}_{2} \mathrm{O}$ ) in the presence of 2 (5 mol\%) under reflux conditions. As summarized in Table 2 (entries 9-12), no solvent afforded better yield than using neat conditions even running the reaction for $2 \mathrm{~h}$ (entry 8 ).

Table 2. Synthesis of $\mathbf{3 a}$ under neat conditions. Catalyst loading, temperature and solvent optimization.

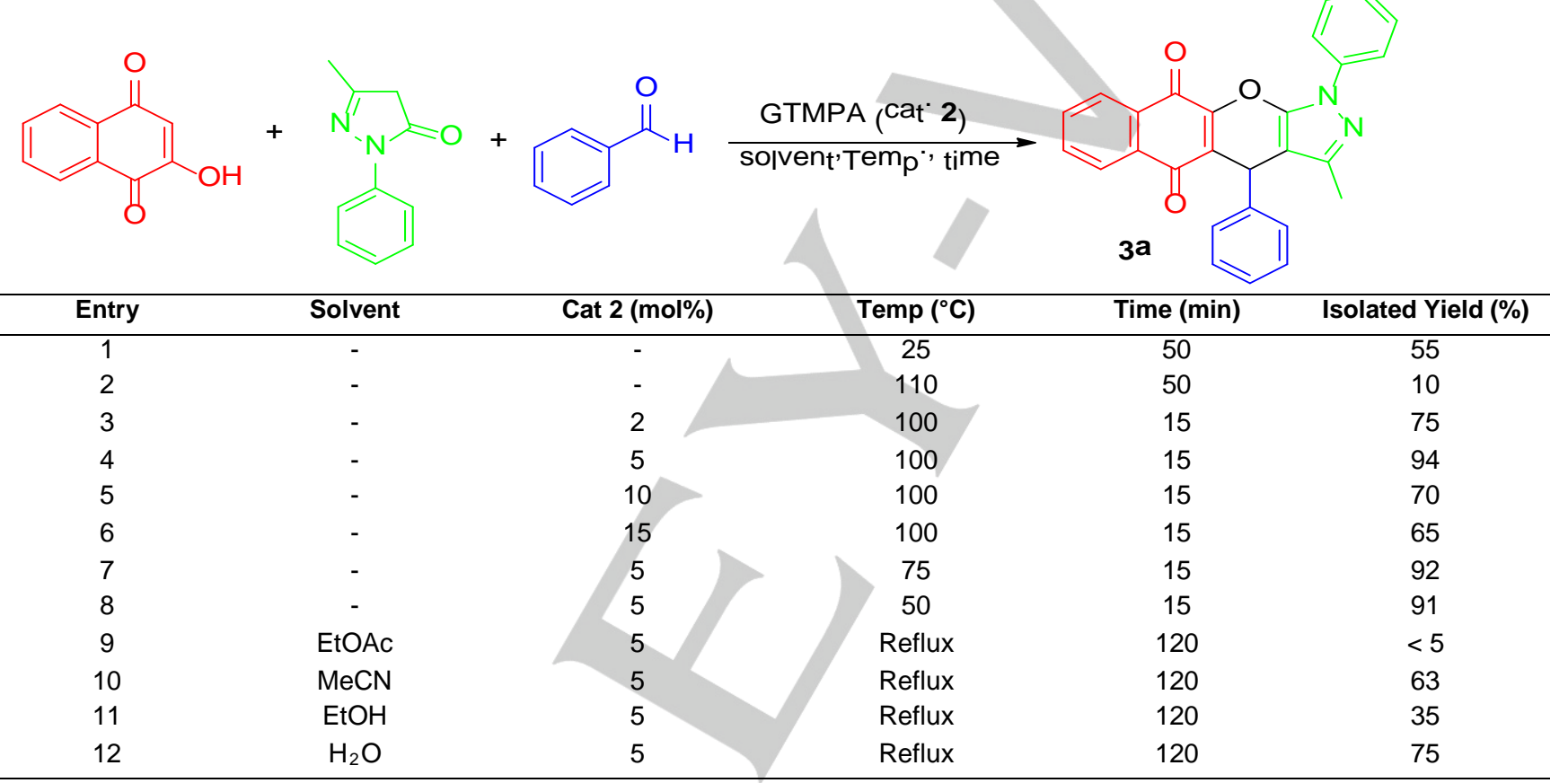

After optimization of the reaction conditions, the target products, as demonstrated with the synthesis of products efficiency and applicability of the method were studied by (Scheme 2). reaction of 2-hydroxynaphthalene-1,4-dione and 3-methyl-1phenyl-1H-pyrazol-5(4H)-one with various aromatic aldehydes in the presence of GTMPA (5 mol\%) under neat conditions. Table 3 summarizes the results which demonstrates the wide scope of the methodology since all the tested functionalized aldehydes (neutral, electron-rich and electron-poor) afforded the desired 3methyl-1,4-diphenyl-1,4-dihydrobenzo[6,7]chromeno[2,3-

c]pyrazole-5,10-dione derivatives in high to excellent yields (86$95 \%$ ) in very short reaction times (5-14 min). Interestingly, the tetra component version of the reaction (see experimental Scheme 2. Synthesis of 3-methyl-1,4-diphenyl-1,4dihydrobenzo[6,7]chromeno[2,3-c]pyrazole-5,10-dione using 2. section, Method B), with the in situ generation of 3-methyl-1phenyl- $1 H$-pyrazol-5(4H)-one from phenylhydrazine and ethyl acetoacetate, was also efficient towards the synthesis of the 
Table 3. Synthesis of 3-methyl-1,4-diphenyl-1,4 dihydrobenzo[6,7]chromeno[2,3-c]pyrazole-5,10-dione derivatives.<smiles>O=C1C=C(O)C(=O)c2ccccc21</smiles><smiles>CC1=NN(c2ccc(C)cc2)C(=O)C1</smiles><smiles>[X]c1ccc(C=O)cc1</smiles>

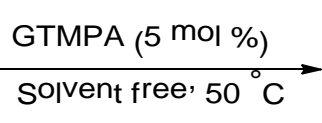<smiles>[X]c1ccc(C2C3=C(Oc4c2c(C)nn4C(=C)C)C(=O)c2ccccc2C3=O)cc1</smiles>

\begin{tabular}{|c|c|c|c|c|c|}
\hline Entry & Aldehyde & & Time (min) A/B & Yield (\%) A/B & M.P. $\left({ }^{\circ} \mathrm{C}\right)$ \\
\hline 1 & Benzaldehyde & $3 a$ & $7 / 9$ & $91 / 88$ & $146-148$ \\
\hline 2 & 4-Chlorobenzaldehyde & $3 b$ & $5 / 6$ & $95 / 92$ & $148-150$ \\
\hline 3 & 2-Chlorobenzaldehyde & $3 c$ & $6 / 7$ & $92 / 90$ & $247-249$ \\
\hline 4 & 3-Nitrobenzaldehyde & $3 d$ & $6 / 9$ & $93 / 91$ & $245-247$ \\
\hline 5 & 4-Methylbenzaldehyde & $3 e$ & $9 / 13$ & $89 / 86$ & $239-241$ \\
\hline 6 & 2-Hydroxybenzaldehyde & $3 f$ & $11 / 13$ & $88 / 86$ & $207-209$ \\
\hline 7 & 3-Hydroxybenzaldehyde & $3 g$ & $9 / 12$ & $89 / 85$ & $240-242$ \\
\hline 8 & 4-Nitrobenzaldehyde & $3 \mathrm{~h}$ & $5 / 6$ & 96/95 & $235-237$ \\
\hline 9 & 2,4-Dichlorobenzaldehyde & $3 \mathbf{i}$ & $5 / 7$ & $92 / 90$ & $247-249$ \\
\hline 10 & 4-Fluorobenzaldehyde & $3 \mathbf{j}$ & $6 / 8$ & $93 / 91$ & $238-240$ \\
\hline 11 & 4-Methoxybenzaldehyde & $3 \mathbf{k}$ & $11 / 14$ & $89 / 87$ & $228-230$ \\
\hline 12 & 2-Hydroxy-1-naphthaldehyde & $3 \mathbf{I}$ & $13 / 17$ & $88 / 84$ & $247-249$ \\
\hline 13 & Terephthalaldehyde & $3 m$ & $10 / 15$ & $92 / 89$ & $210-212$ \\
\hline 14 & 2,6-Difluorobenzaldehyde & $3 n$ & $7 / 9$ & $90 / 92$ & $247-249$ \\
\hline 15 & 4-(Trifluoromethyl) benzaldehyde & 30 & $10 / 12$ & $90 / 91$ & $210-212$ \\
\hline 16 & 4-Formylbenzonitrile & $3 p$ & $11 / 13$ & $92 / 90$ & $228-230$ \\
\hline 17 & 3,5-Difluorobenzaldehyde & $3 q$ & $10 / 11$ & $90 / 89$ & $226-228$ \\
\hline 18 & Thiophene-2-carbaldehyde & $3 r$ & $15 / 19$ & $82 / 77$ & $230-232$ \\
\hline 19 & Furan-2-carbaldehyde & 3s & $18 / 23$ & $81 / 76$ & $210-213$ \\
\hline
\end{tabular}

A catalytic cycle proposal for the multicomponent synthesis of dihydrobenzo[6,7]chromeno[2,3-c] 3-methyl-1,4-diphenyl-1,4pyrazole-5,10-dione derivatives is shown in Scheme 3. As shown, the aldehyde is initially activated by GTMPA towards the nucleophilic attack of 2hydroxynaphthalene-1,4-dione affording, after dehydration, the 3-methylenenaphthalene intermediate I. Then, 5-methyl-2phenylpyrazolidin-3-one (obtained from ethyl acetoacetate and phenyl hydrazine following Method B) reacts via conjugate addition with I to afford adduct II, which suffers an intramolecular cyclization/dehydration process assisted by $\mathbf{2}$ to give intermediate III. A final 2-assisted deprotonation of III yields the reaction product.

The recyclability and reuse of the catalyst was also studied on the reaction of benzaldehyde ( $1 \mathrm{mmol})$, 2hydroxynaphthalene-1,4-dione ( $1 \mathrm{mmol})$ and 3-methyl-1-phenyl$1 \mathrm{H}$-pyrazol-5(4H)-one $(1 \mathrm{mmol})$ at $50{ }^{\circ} \mathrm{C}$. After completion the reaction as monitored by TLC, EtOAc $(10 \mathrm{~mL})$ was added to the reaction mixture, the catalyst was separated from solution using a centrifugation (1000 rpm). The remained catalyst was washed with hot acetone $(10 \mathrm{~mL})$, dried and was used for the next runs. As indicated in Fig. 9, catalyst could be recycled and efficiently reused up to 8 reaction cycles with no detectable loss of catalytic activity.

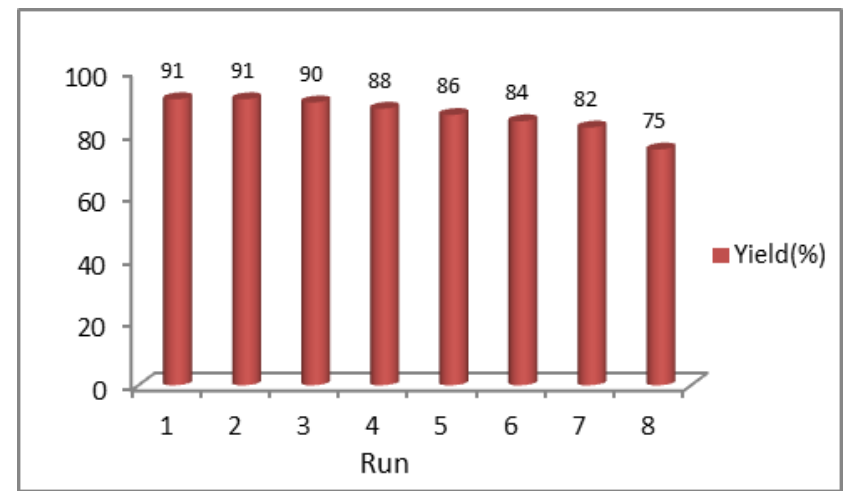

Fig. 9. Recyclability of 2 as catalyst in the synthesis of 3-methyl-1,4 diphenyl-1,4-dihydrobenzo[6,7]chromeno[2,3-c] pyrazole-5,10-dione under solvent free condition at $50{ }^{\circ} \mathrm{C}$. Reaction time $8 \mathrm{~min}$. 


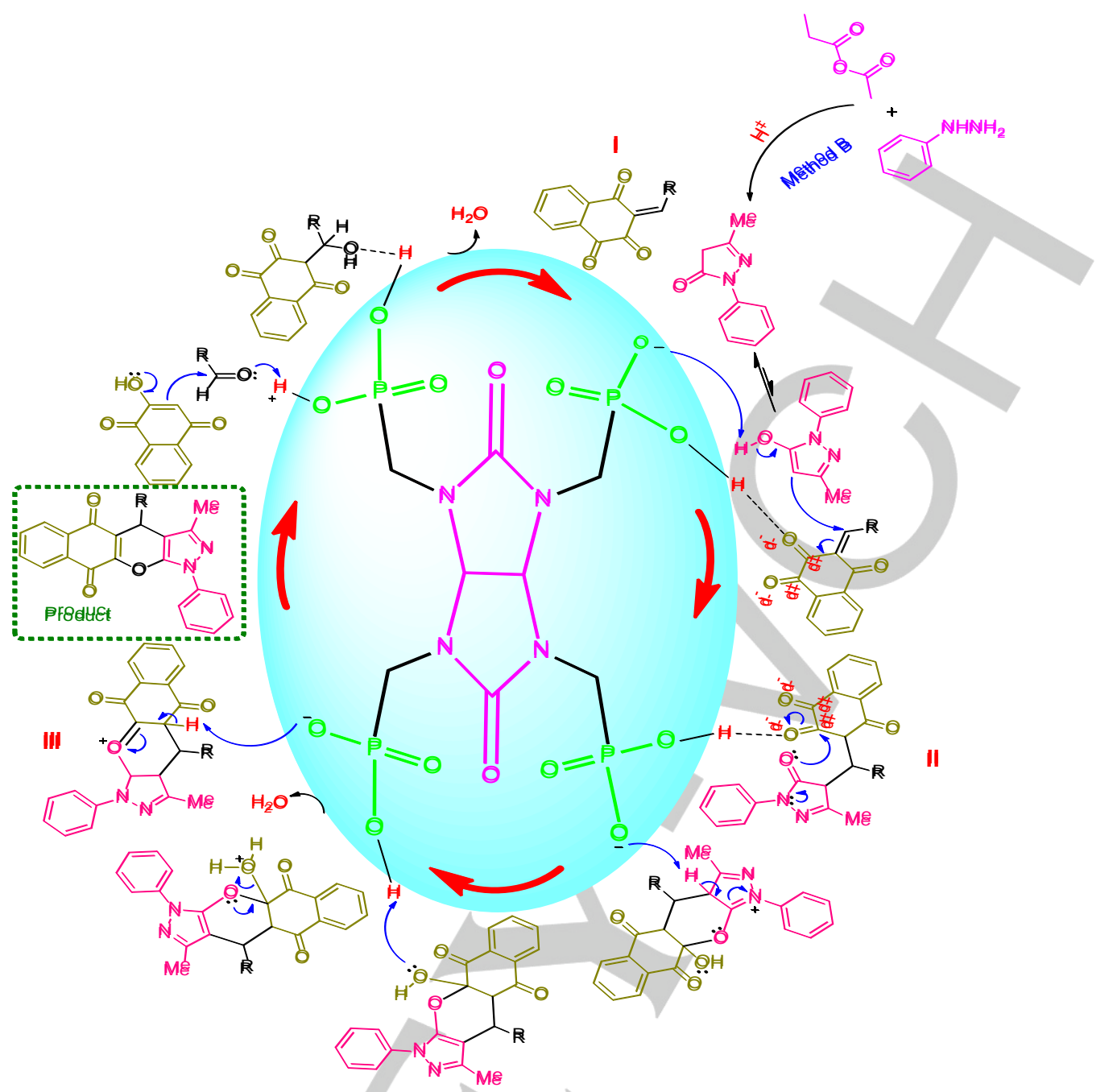

Scheme 3. Mechanism for the 2-catalyzed synthesis of 3-methyl-1,4-diphenyl-1,4-dihydrobenzo[6,7]chromeno[2,3-c]pyrazole-5,10-diones under solvent free conditions

\section{Conclusions}

In conclusion, herein we present the praparation of a crabby biological-based glycoluril tetrakis(methylene phosphorous acid) (GTMPA) for the first time. Two convenient methods for the synthesis of novel biological henna-based 3methyl-1,4-diphenyl-1,4-dihydrobenzo[6,7]chromeno[2,3-

c]pyrazole-5,10-diones were also reported in the presence of described nano glycoluril tetrakis(methylene phosphorous acid) (GTMPA) as an efficient and recyclable catalyst under neat conditions. The major advantages of the presented method are high yields, short reaction times, and the reusability of the catalyst. We think that the present work can open up a new and promising insight in the course of rational design, synthesis and application of other biological-based, task-specific and advanced nano materials with phosphorous acid tags for various purposes.

The described biological-based glycoluril with phosphorous acid pending may be useful for the synthesis of gels, gelators, selfhealing and smart hydrogels. Further systematic research for knowledge-based development of this research field is going on in our research group.

\section{Acknowledgements}

We thank Bu-Ali Sina University, Iran National Science Foundation (INSF) (Grant Number: 96003376), National Elites Foundation, University of Alicante (VIGROB-173, UAUSTI16-03), and the Spanish Ministerio de Economía y Competitividad (CTQ2015-66624-P) for financial support to our research groups.

Keywords: Glycoluril, Glycoluril tetrakis(methylene phosphorous acid) (GTMPA), Biological-based, Nanostructured catalyst, 
Recyclable catalyst, 3-Methyl-1,4-diphenyl-1,4dihydrobenzo[6,7]chromeno[2,3-c]pyrazole-5,10-dione.

[1] P. T. Anastas, J. B. Zimmerman, Green Chem. 2016, 18, 43244324.

[2] P. T. Anastas, J. C. Warner, Green Chemistry: Theory and Practice, Oxford University Press: New York, 1998.

[3] G. Mohammadi Ziarani, N. Lashgari, A. Badiei, J. Mol. Catal. A Chem. 2015, 397, 166-191.

[4] M. Kaur, S. Sharma, P. M. S. Bedi, Chin. J. Catal. 2015, 36, 520 549.

[5] F. Su, Y. Guo, Green Chem. 2014, 16, 2943-2957.

[6] Y. M. Sani, W. M. A. W. Daud, A. R. Abdul Aziz, Appl. Catal. A: Gen. 2014, 470, 140-161.

[7] R. M. N. Kalla, H. Park, T. T. K. Hoang, I. Kim, Tetrahedron Lett 2014, 55, 5373-5376.

[8] W. Xie, H. Wang, H. Li, Ind. Eng. Chem. Res. 2012, 51, 225-231.

[9] M. A. Zolfigol, V. Khakyzadeh, A. R. Moosavi-Zare, A. Rostami, A. Zare, N. Iranpoor, M. H. Beyzavi, R. Luque, Green Chem. 2013 15, 2132-2140.

[10] M. Daraei, M. A. Zolfigol, F. Derakhshan-Panah, M. Shiri, H. G. Kruger, M . Mokhleshi, J. Iran. Chem. Soc. 2015, 12, 855-861.

[11] M. Mokhtary, J. Iran. Chem. Soc. 2016, 13, 1827-1845.

[12] M. A. Zolfigol, H. Ghaderi, S. Bagheri, L. Mohammadi, J. Iran. Chem. Soc. 2017, 14, 121-134.

[13] V. Polshettiwar, R. S. Varma, Green Chem. 2010, 12, 743-754.

[14] F. Cao, P. Yin, X. Liu, C. Liu, R. Qu, Renew. Energy 2014, 71, 61 68.

[15] R. V. A. Orru, M. D. Greef, Synthesis 2003, 10, 1471-1499.

[16] V. Nair, C. Rajesh, A. U. Vinod, S. Bindu, A. R. Sreekanth, J. S Mathen, L. Balgopal, Acc. Chem. Res. 2003, 36, 899-907.

[17] L. F. Tietze, A. Modi, Med. Chem. Res. 2000, 20, 304-322.

[18] E. Abbaspour-Gilandeh, M. Aghaei-Hashjin, P. Jahanshahi, M. S. Hoseininezhad-Namin, Monatsh Chem. 2017, 148, 731-738.

[19] F. Al-Assar, K. N. Zelenin, E. E. Lesiovskaya, I. P. Bezhan, B. A Chakchir, Pharm. Chem. 2002, 36, 598-603.

[20] R. P. Jain, J. C. Vederas, Bioorg. Med. Chem. Lett. 2004, 14 3655-3658.
[21] S. Kumar, H. Ila, H. Junjappa, J. Org. Chem. 2009, 74, 7046-7051.

[22] M. Li, Y.-L. Hou, L.-R. Wen, F.- M. Gong, J. Org. Chem. 2010, 75 8522-8532.

[23] S. Rostamnia, A. Hassankhani, RSC Adv. 2013, 3, 18626-18629.

[24] R. H. Thomson, Naturally occurring quinones. 4th ed. London, Chapman \& Hall, 1997.

[25] S. Subramanian, M. M. C. Ferreira, M. Trsic, J. Struct. Chem. 1998 , 9, 47-57.

[26] A. F. Santos, P. A. L. Ferraz, A. V. Pinto, M. C. F. R. Pinto, M. O. F. Goulart, A. E. G. Sant'Ana, Int. J. Parasitol 2000, 30,1199-1202.

[27] M. J. Teixeira, Y. M. De Almeida, J. R. Viana, J. G. Holanda Filha T. P. Rodrigues, J. R. C. Prata Jr, Phytother. Res. 2001, 15, 44-48.

[28] E. R. De Almedia, A. A. D. S. Filho, E. R. D. Santos, C. A. C. Lopes, J. Ethnopharmacol 1990, 29, 239-241.

[29] S. Garnier, J. L. Wolfender, M. Nianga, H. Stoeckli-Evans, K. Hostettmann, Phytochemistry 1996, 42, 1315-1320.

[30] B. S. Siddiqui, M. N. Kardar, T. Ali, S. Khan, Helv. Chim. Acta. 2003, 86: 2164-2169.

[31] A. L. Perez, G. Lamoureux, A. Sanchez-Kopper, Tetrahedron Lett. 2007, 48, 3735-3738

[32] M. Dabiri, Z. Noroozi Tisseh, A. Bazgir, Dyes and Pigments 2011 , 89: 63-69.

[33] V. K. Tandon, H. K. Maurya, Tetrahedron Lett. 2009, 50, 58965902

[34] V. K. Tandon, H. K. Maurya, Tetrahedron Lett. 2010, 51, 3843 3847.

[35] H. B. Zhang, L. Liu, Y. J. Chen, D. Wang, C. J. Li, Eur. J. Org Chem. 2006, 869-873.

[36] a) A. Yaghoubi, M. G. Dekamin, Chem. Select. 2017, 2, 9236 9243; b) K. I. Assaf, W. M. Nau, Chem. Soc. Rev. 2015, 44, 394 418; c) J. Mohanty, Encyclopedia of Polymer Science and Technology, DOI: 10.1002/0471440264.pst639; d) P. I. Dalko, L. Moisan, Angew. Chem. Int. Ed. 2001, 40, 3726-3748; e) P. I. Dalko , L. Moisan, Angew. Chem. Int. Ed. 2004, 116, 5248-5286; f) C. Bolm, Adv. Synth. Catal. 2004, 346, 1022-1022; g) M. G. Dekamin, S. Ilkhanizadeh, Z. Latifidoost, H. Daemi, Z. Karimi, M. Barikani, RSC Adv. 2014, 4, 56658-56664.

[37] L. N. Bùi, M. Thompson, N. B McKeown, A. D. Romaschin and P. G. Kalman, Analyst 1993, 118, 463-474.

[38] P. Y. Shih, S. W. Yung, T. S. Chin, J. non-Cristalline Solids 1999 244, 211-222. 


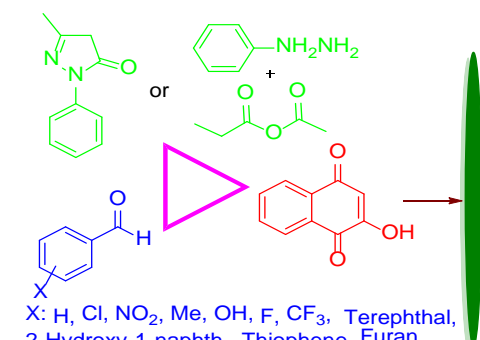

$\mathrm{X}: \mathrm{H}, \mathrm{Cl}, \mathrm{NO}_{2}, \mathrm{Me}, \mathrm{OH}, \mathrm{F}, \mathrm{CF}_{3}$, Terephth
2-Hydroxy-1-naphth, Thiophene, Furan

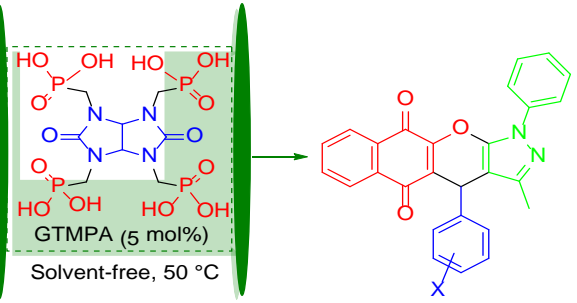

Saeid Moradi, ${ }^{a}$

Mohammad Ali

Zolfigol, ${ }^{* a}$

Mahmoud Zarei, *a

Diego A. Alonso, ${ }^{{ }^{b}}$

Abbas

Khoshnood ${ }^{\star b}$

Page No. - Page No.

Title 
Additional Author information for the electronic version of the article.

${ }^{\mathrm{a} D e p a r t m e n t ~ o f ~ O r g a n i c ~ C h e m i s t r y, ~ F a c u l t y ~ o f ~ C h e m i s t r y, ~ B u-A l i ~ S i n a ~ U n i v e r s i t y, ~ H a m e d a n ~ 6517838683, ~ T e l: ~+988138282807, ~ F a x: ~+988138380709 ~ I r a n . ~ E-M a i l: ~}$ zolfi@basu.ac.ir \& mzolfigol@yahoo.com (Mohammad Ali Zolfigol), mahmoud8103@yahoo.com (Mahmoud Zarei)

${ }^{\text {b} O r g a n i c ~ S y n t h e s i s ~ I n s t i t u t e ~ a n d ~ O r g a n i c ~ C h e m i s t r y ~ D e p a r t m e n t, ~ A l i c a n t e ~ U n i v e r s i t y, ~ A p d o . ~ 99, ~} 03080$ Alicante, Spain. E-Mail: diego.alonso@ua.es (Diego A. Alonso), abbas.khoshnood@ua.es (Abbas Khoshnood) 